\title{
Transmission Line Based Metamaterials for Acoustic Waves
}

\author{
$\underline{\text { Frédéric Bongard }}^{1}$, Hervé Lissek ${ }^{2}$, and Juan R. Mosig ${ }^{2}$ \\ ${ }^{1}$ JAST SA Antenna Systems, PSE-EPFL Bat. C, CH-1015 Lausanne, Switzerland, frederic.bongard@a3.epfl.ch \\ ${ }^{2}$ Laboratory of Electromagnetics and Acoustics (LEMA), Ecole Polytechnique Fédérale de Lausanne (EPFL), CH- \\ 1015 Lausanne, Switzerland, herve.lissek@epfl.ch, juan.mosig@epfl.ch
}

\begin{abstract}
We present our recent work on a one-dimensional acoustic negative refractive index metamaterial based on the concept of dual transmission line extensively investigated in microwave engineering. The proposed structure consists of an acoustic waveguide periodically loaded with membranes realizing the function of series "capacitances" and transversally connected open channels realizing shunt "inductances". It exhibits a negative refractive index band over almost one octave, from 0.6 to $1 \mathrm{kHz}$. Using formal analogies, we describe how simple acoustic circuit models can be used for efficient design of metamaterials both in terms of dispersion and impedance.
\end{abstract}

\section{Introduction}

After an extensive development in the area of electromagnetics and optics [1-3], acoustic metamaterials have recently received considerable interest. The latter consist of composite structures obtained by loading a host fluid medium with subwavelength elastic and/or fluid inclusions, for which effective parameters such as the mass density and the bulk modulus (the counterparts of $\varepsilon$ and $\mu$ for electromagnetic waves) can be defined and possibly take negative values, thereby enabling new phenomena such as acoustic negative refraction. In the realm of electromagnetics, there is a common distinction between two types of metamaterials: arrays of resonant inclusions, such as the split-ring resonator and wire medium, and transmission line (TL) based metamaterials [2, 3]. While the materials of the first kind are inherently narrow band and lossy due to their resonant nature, the latter can exhibit the desired meta-properties, such as negative refraction, over a much larger bandwidth and with lower losses since they do not explicitly rely on resonance phenomena. It appears that most of the acoustic metamaterials with negative parameters reported to date are based on localized resonances in the unit cell, hence the name "locally resonant sonic material", and thus belong to the first category mentioned above [4-6].

It has been only recently that the acoustic counterparts of TL-based metamaterials have been reported [7, 8]. These structures require the realization of acoustic or mechanical elements which implement shunt "inductances" (i.e., acoustic masses) and series "capacitances" (i.e., acoustic compliances). This paper reviews our work on the topic, in the light of formal analogies between electromagnetics and acoustics through an extensive use of circuit modeling, which is shown to be a powerful tool for both understanding and design purposes.

\section{Transmission Line Modeling and Metamaterials}

TLs are circuit-based concepts that describe the propagation of waves in different systems. In acoustic circuit modeling, the acoustic pressure $p$ can substitute for the electric voltage, and the volume velocity $q$ flowing through a surface $S$ substitutes for the electric current, According to this convention, an incremental section of a conventional fluid can be described by the model of Figure 1(a), where $m_{\mathrm{a}}$ is an acoustic mass (or inertance) and $C_{\mathrm{a}}$ is an acoustic compliance, and $\rho$ and $K$ are the density and bulk modulus of the medium (here air), respectively. The corresponding wave velocity is given by $c=\sqrt{K / \rho} \cong 340 \mathrm{~m} / \mathrm{s}$.

Figure 1(b) shows the dual topology of the conventional TL, which exhibits a negative refractive index over an infinite bandwidth. In practice, it is realized by periodically loading a host medium with discrete shunt acoustic masses and series acoustic compliances, resulting in the so-called composite right/left-handed transmission line (CRLH TL) [2]. This paper describes a possible realization of the circuit shown in Figure 1(c) for acoustic waves. 


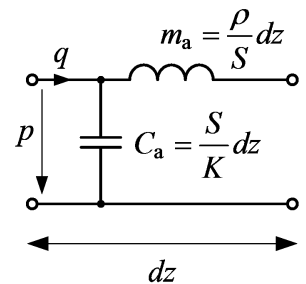

(a)

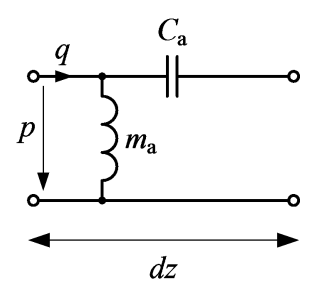

(b)

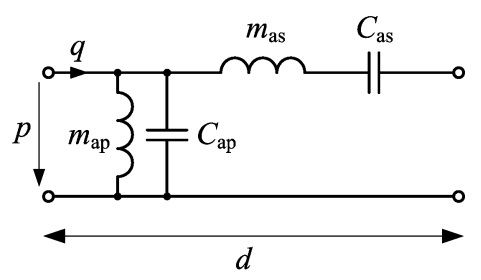

(c)

Figure 1: Incremental circuit for (a) a conventional medium and (b) a dual medium exhibiting a negative refractive index. (c) Unit cell of the CRLH TL of lattice constant $d$.

It should be pointed out that, in our opinion, the main feature of TL based metamaterials is not that they can be described by circuit and TL models (arrays of resonant particles in free space can also be described in such a way), but rather that they are physical structures designed with the goal of implementing the dual TL concept (or more generally the CRLH TL), and hence exhibit negative parameters over a much wider bandwidth than their resonant counterparts.

\section{Description and design of the proposed structure}

The proposed CRLH metamaterial implementation is shown in Figure 2(a). The host "medium" is an acoustic waveguide with circular cross-section, the radius of which is $a=9.06 \mathrm{~mm}$, and perfectly rigid walls. The lattice constant is $d=34 \mathrm{~mm}$, corresponding to one tenth of the wavelength at the central frequency of $1 \mathrm{kHz}$, which justifies an effective medium description.

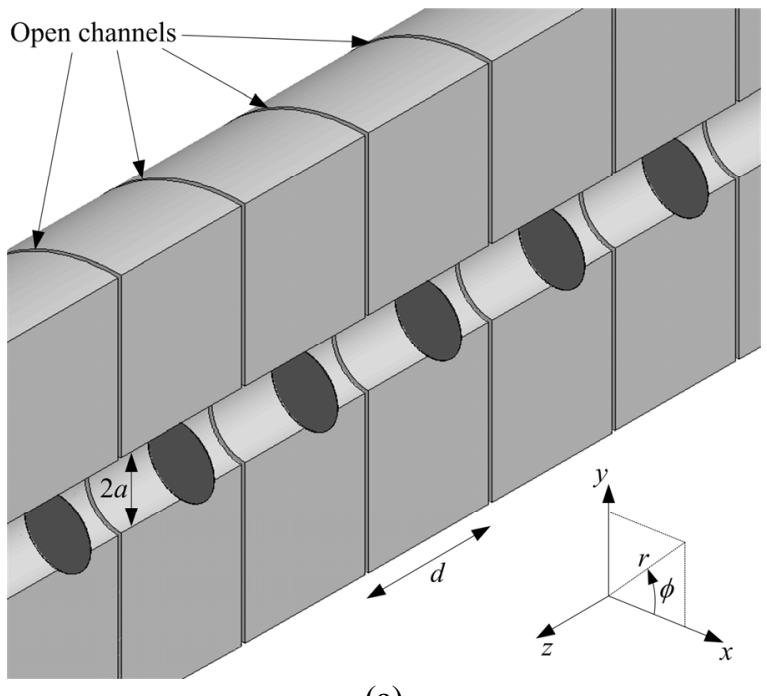

(a)

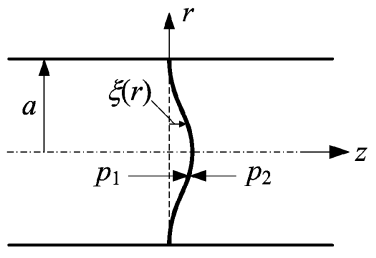

(b)

$\Delta^{r}$

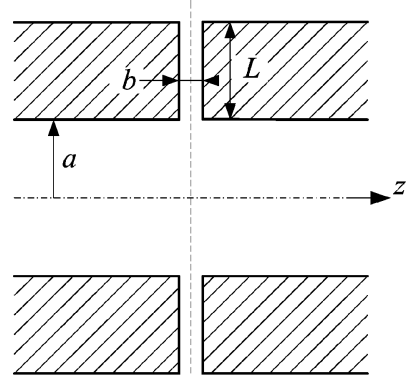

(d)

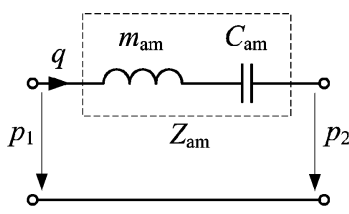

(c)

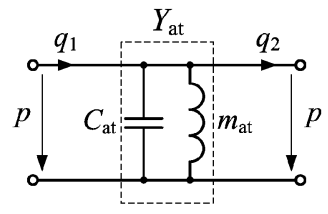

(e)

Figure 2: (a) Proposed CRLH TL combining membranes (in dark) and radial open channels. The main pipe has been cut in the $y z$ plane for visibility. (b) Elastic circular membrane clamped to an acoustic waveguide with its equivalent acoustic circuit (c). (d) Shorted radial stub connected to an acoustic circular waveguide with its equivalent acoustic circuit (e).

The series compliances are realized with $125 \mu \mathrm{m}$ thick Kapton membranes clamped at their perimeter to the host waveguide [Figure 2(b)]. For such a loading element, a difference of pressure exists across the membrane, while the volume velocity remains constant, thus corresponding to a series impedance. The restoring force of the membrane provides the required series compliance $C_{\mathrm{am}}$, while its physical mass, considered here as a parasitic, is accounted for in the acoustic domain by an acoustic mass $m_{\mathrm{am}}$, resulting in the circuit model of Figure 2(c). 
Shunt acoustic masses can be realized with short open tubes transversally connected to the main host waveguide. Such structures are often referred to as "stubs" in microwave engineering. A physically open tube is characterized by an almost zero pressure at its termination, which corresponds to a short-circuited stub. Based on this concept, the solution adopted here consists of radial channels opened in the main waveguide structure, as shown in Figure 2(a) and (d). For such a loading element, a certain amount of volume velocity is deviated into the radial stub, while the pressure remains constant across it, thus corresponding to a shunt admittance as shown in Figure 2(e). Further details on the modeling and design of membranes and acoustic radial stubs can be found in [7].

\section{Performances}

The proposed TL-based metamaterial has been designed using the presented equivalent circuits and its performances have been assessed by full-wave simulations using the FEM solver COMSOL MULTIPHYSICS. In the simulation setup, one or several unit cells are characterized in terms of reflection and transmission coefficients under plane wave incidence, from which the properties of the periodic structure can be deduced. The relevant parameters are the Bloch propagation constant (or dispersion diagram) and the Bloch impedance, which is of interest for matching the periodic structure with other devices [9].

The Bloch parameters for the considered metamaterial are shown in Figure 3, for both simulation and circuit model results [Figure 1(c)]. It can be observed on the dispersion diagram of Figure 3(a) that the structure exhibits a negative refractive index band (opposite phase and group velocities) over a 50\% relative bandwidth, from the LH cutoff $f_{\mathrm{cL}}=0.61 \mathrm{kHz}$ to the transition frequency $f_{0}=1 \mathrm{kHz}$, and a positive refractive index band from $f_{0}$ to the RH cutoff $f_{\mathrm{cR}}=1.64 \mathrm{kHz}$. There is no band gap between these two bands (balanced condition), which results in a frequency with zero phase constant (and thus zero refractive index) and non-zero group velocity. A very good agreement is observed between full-wave and circuit model results.

The Bloch impedance around the transition frequency $f_{0}$ is smoothly varying with frequency, which is favorable for wideband matching to an external waveguide (here with radius $5.5 \mathrm{~mm}$ ). Simulation of a ten cell long structure confirms the good matching of the design [7].

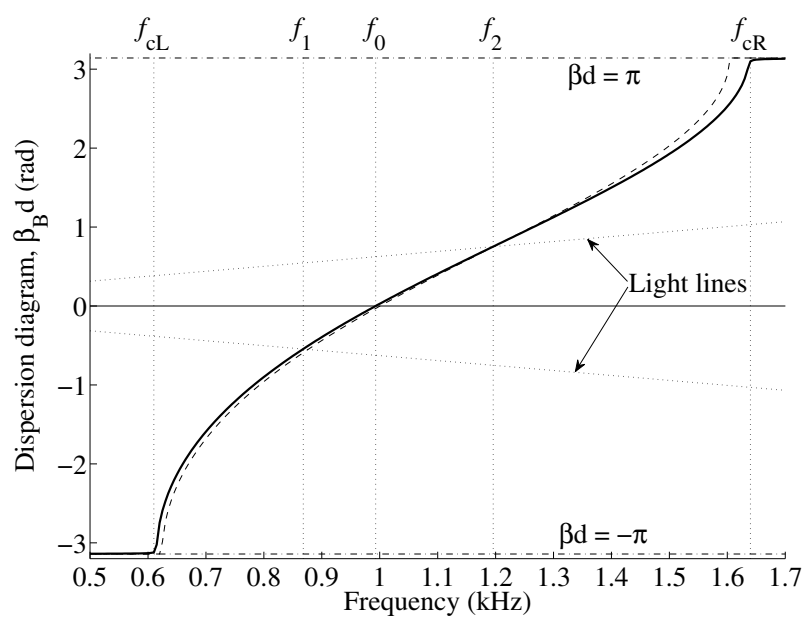

(a)

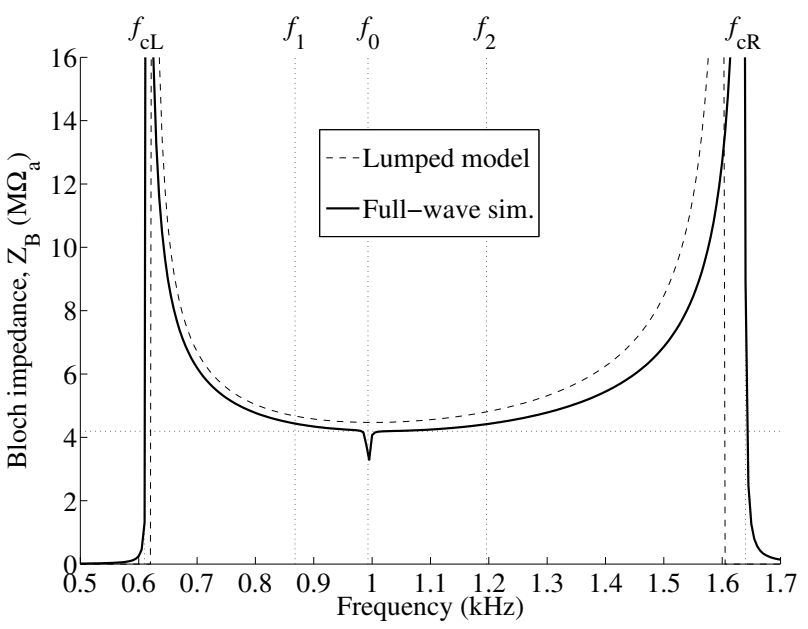

(b)

Figure 3: Bloch parameters extracted from full-wave simulation results (COMSOL MULTIPHYSICS) and circuit models for the implemented CRLH TL. $f_{\mathrm{cL}}$ and $f_{\mathrm{cR}}$ are the LH and RH cutoff frequencies, respectively, $f_{0}$ is the transition frequency between the LH and RH bands, and $f_{1}$ and $f_{2}$ are the limits of the fast-wave band. The "light lines" correspond to the dispersion in air, by analogy with the terminology used in photonic crystals.

Another interesting property of this periodic structure is the radiation occurring in the fast-wave band of the dispersion diagram (between $f_{1}$ and $f_{2}$ in Figure 3 ). This peculiar feature for this type of metamaterials has been extensively exploited in microwave antenna engineering for the development of a novel type of leaky-wave antennas with full-space scanning capabilities [2,3]. The proposed structure thus represents the analogue for acoustic waves 
of these antennas, thereby paving the way for the investigation of novel directive acoustic sensors or sources based on this principle.

\section{Conclusion}

Circuit theory concepts have been efficiently used to conceptualize and design an acoustic non-resonant TL-based metamaterial, using membranes and shunt acoustic stubs. The structure presents a negative refractive index over almost one octave $(0.6$ to $1 \mathrm{kHz})$ and a zero refractive index at the seamless transition between the negative and positive index bands. The developed structure might find applications in subwavelength resonant cavities based on phase compensation between negative and positive index media. Further work should aim at the investigation of two-dimensional structures based on the same principle. Here, targeted applications are wideband and well-matched negative index acoustic lenses for acoustic imaging applications or cloaking devices.

\section{Acknowledgments}

This work was supported by the Swiss National Scientific Foundation under research grant 200021-130255.

\section{References}

1. N. Engheta and R. W. Ziolkowski, Metamaterials: Physics and Engineering Explorations: Wiley-Interscience and IEEE press, 2006.

2. C. Caloz and T. Itoh, Electromagnetic Metamaterials: Transmission Line Theory and Microwave Applications: Wiley-Interscience and IEEE press, 2006.

3. G. V. Eleftheriades and K. G. Balmain, Negative-Refraction Metamaterials: Fundamental Principles and Applications: Wiley-Interscience and IEEE press, 2005.

4. L. Fok, M. Ambati, and X. Zhang, "Acoustic Metamaterials," MRS Bulletin, vol. 33, October 2008, pp. 931-934.

5. Z. Liu, X. Zhang, Y. Mao, Y. Y. Zhu, Z. Yang, C. T. Chan, and P. Sheng, "Locally Resonant Sonic Materials," Science, vol. 289, September 2000, pp. 1734-1736.

6. N. Fang, D. Xi, J. Xu, M. Ambati, W. Srituravanich, C. Sun, and X. Zhang, "Ultrasonic metamaterials with negative modulus," Nature Materials, vol. 5, June 2006, pp. 452-456.

7. F. Bongard, H. Lissek, and J. Mosig, "Acoustic transmission line metamaterial with negative/zero/positive refractive index," physical Review B, vol. 82, 16 September 2010, p. 094306.

8. S. H. Lee, C. M. Park, Y. M. Seo, Z. G. Wang, and C. K. Kim, "Composite acoustic medium with simultaneously negative density and modulus," Physical Review Letters, vol. 104, February 2010, p. 054301.

9. D. M. Pozar, Microwave Engineering: Addison-Wesley Publishing Company, Inc., 1990. 